\title{
EEG and MEG: Forward Solutions for Inverse Methods
}

\author{
John C. Mosher, Richard M. Leahy,* and Paul S. Lewis
}

\begin{abstract}
A solution of the forward problem is an important component of any method for computing the spatio-temporal activity of the neural sources of magnetoencephalography (MEG) and electroencephalography (EEG) data. The forward problem involves computing the scalp potentials or external magnetic field at a finite set of sensor locations for a putative source configuration. We present a unified treatment of analytical and numerical solutions of the forward problem in a form suitable for use in inverse methods. This formulation is achieved through factorization of the lead field into the product of the moment of the elemental current dipole source with a "kernel matrix" that depends on the head geometry and source and sensor locations, and a "sensor matrix" that models sensor orientation and gradiometer effects in MEG and differential measurements in EEG. Using this formulation and a recently developed approximation formula for EEG, based on the "Berg parameters," we present novel reformulations of the basic EEG and MEG kernels that dispel the myth that EEG is inherently more complicated to calculate than MEG. We also present novel investigations of different boundary element methods (BEM's) and present evidence that improvements over currently published BEM methods can be realized using alternative error-weighting methods. Explicit expressions for the matrix kernels for MEG and EEG for spherical and realistic head geometries are included.
\end{abstract}

Index Terms - Boundary element method (BEM), electroencephalogram (EEG), forward model, head modeling, realistic head model, spherical head model.

\section{INTRODUCTION}

$\mathbf{N}$ EURAL current sources in the brain produce external magnetic fields and scalp surface potentials that can be measured using magnetoencephalography (MEG) and electroencephalography (EEG), respectively. The current fields in the head that produce these EEG and MEG (collectively E/MEG) signals can be separated into two components, the primary current term (cf. [49]), representing the impressed neural and microscopic passive cellular currents, and the secondary or volume currents that are a result of the macroscopic electric field. The primary currents are considered to be the sources of interest in E/MEG, since they represent the areas of neural activity associated with a given sensory, motor, or

Manuscript received October 3, 1997; revised July 22, 1998. This work was supported by the National Institute of Mental Health under Grant R01-MH53213, and by Los Alamos National Laboratory, operated by the University of California for the United States Department of Energy, under Contract W-7405-ENG-36. Asterisk indicates corresponding author.

J. C. Mosher is with the Los Alamos National Laboratory, Los Alamos, NM 87545 USA.

*R. M. Leahy is with the Signal and Image Processing Institute, University of Southern California, Los Angeles, CA 90089-2564 USA (e-mail: leahy@sipi.usc.edu).

P. S. Lewis is with the Los Alamos National Laboratory, Los Alamos, NM 87545 USA.

Publisher Item Identifier S 0018-9294(99)01841-8. cognitive process. The recent development of systems with whole-head coverage offer the potential for E/MEG to produce accurate estimates of the location and time courses of these underlying primary sources. In the context of the localization of neural sources, the forward problem is then to determine the potentials and magnetic fields that result from primary current sources. The inverse problem is to estimate the location of these primary current sources.

The emphasis in E/MEG modeling is, therefore, the relationship between a primary current source distribution and the data at the sensor array. As reviewed by Tripp [49], the linearity of the forward model can be expressed as the inner product of a vector lead field [6] and the primary current. Since the majority of inverse methods for E/MEG are based on linear algebraic formulations, a matrix formulation is a natural framework for the solution of the forward problem. To simplify the presentation here, we restrict the primary current to current dipoles, since more complicated sources can be expressed as sums or integrals of these elemental sources. We describe solutions to the forward problem for both MEG and EEG by partitioning the lead field into the product of a sensor matrix, a kernel matrix and the moment of the dipole. The sensor matrix models sensor orientation and gradiometer effects in MEG and the effects of the reference electrode(s) in EEG. The kernel matrices are functions only of the sensor and source locations and the head geometry. This form, in which the dipole moment is explicitly factored out of the forward model, is well suited for source localization methods in which the dipole orientations are unconstrained [33] and for studies such as that in [34], where we examine bounds on localization accuracy as a function of dipole location. Furthermore, our explicit matrix formulations of the forward solutions make these results directly applicable to the various linear imaging models, e.g., [12], [27], and [42]. Finally, decoupling of the kernel and sensor matrices facilitates studies of issues such as the effect of the reference electrode in EEG and comparisons of planar and radial gradiometers in MEG.

The most commonly used head model assumes that it is made up of a set of nested concentric spheres, each with homogeneous and isotropic conductivity. Under this assumption, both the EEG and MEG problems admit to wellknown closed form solutions. Here, we describe the forward solutions for both problems for the spherical model using kernel matrices. The matrices are explicitly stated here in Cartesian coordinates. While these results are well known, presentation in this form is novel and suitable for direct use in inverse methods with unconstrained dipole orientations. 
High-resolution anatomical imaging of subjects is becoming routine, and the trend in the biophysics community is to move toward more realistic nonspherical head models. By far the most common approach in E/MEG for arbitrary head geometries is the boundary element method (BEM). Here, we review the E/MEG BEM literature in terms of the method of weighted residuals. We show that the majority of published methods can be viewed in this framework, using either the collocation or Galerkin weighting methods [5], [48]. We include numerical comparisons of collocation and Galerkin methods using linear and constant basis functions and show that a linear Galerkin method, which has not previously been used in this field, can produce substantial improvements in accuracy. We also examine the effect of the "isolated skull approach" (ISA) [26] on the accuracy of the computed fields.

The solutions for the forward EEG and MEG problems for spherical and realistic head geometries are presented here in an attempt to: i) provide a unified framework for comparison of the accuracy and computational cost of different solutions, ii) to present these different solutions in a consistent notation in relatively concise form, and iii) to present the solutions in a form suitable for direct use in inverse procedures and for comparisons of different modalities and system configurations.

The paper is organized as follows. In Section II, we review the basic quasi-static assumptions used in E/MEG, both to establish notation and to clarify where some of the simplifying assumptions arise that allow the BEM approach to solving the forward problem. In Section III, we present the analytical solutions for the spherical head models in a form suitable for generating gain matrices. In Section IV, we develop discrete formulations of the BEM solutions using the method of weighted residuals, then compare the effects of the choice of basis and weighting functions. In Section V, we discuss in particular the issues and implications of the various approaches to BEM, and review several recent publications within the framework presented here.

\section{THE FORWARD PROBLEM}

We review first the assumptions that form the basis of most of the E/MEG forward models. For the biological signals of interest in E/MEG, the time-derivatives of the associated electric and magnetic fields are sufficiently small that they can be ignored in Maxwell's equations. Recent discussions and details of this quasi-static approximation can be found in [27], [28], and [49]. The static magnetic field equations are $\nabla \times \boldsymbol{b}(\boldsymbol{r})=\mu_{0} \boldsymbol{j}(\boldsymbol{r})$ and $\nabla \cdot \boldsymbol{b}(\boldsymbol{r})=0$, i.e., the curl of the magnetic field at location $\boldsymbol{r}$ is proportional to the current density, and the divergence of the magnetic field is zero. We are interested in the current density $\boldsymbol{j}(\boldsymbol{r})$ in a closed volume of finite conductivities. Outside this volume the conductivity and current density are zero. The integral equation relating $\boldsymbol{b}(\boldsymbol{r})$ and $\boldsymbol{j}(\boldsymbol{r})$ is the integral form of the Biot-Savart law

$$
\boldsymbol{b}(\boldsymbol{r})=\frac{\mu_{0}}{4 \pi} \int_{G} \boldsymbol{j}\left(\boldsymbol{r}^{\prime}\right) \times \boldsymbol{d} / d^{3} d \boldsymbol{r}^{\prime}
$$

where $\boldsymbol{d}=\boldsymbol{r}-\boldsymbol{r}^{\prime}$ (with magnitude $d$ ) is the distance between the observation point $\boldsymbol{r}$ and the source point $\boldsymbol{r}^{\prime}$, and the integration is carried out over a closed volume $G$.
We divide the current into two components, passive and primary. We define as passive those currents that are a result of the macroscopic electric field in the conducting medium of the volume $\boldsymbol{j}^{v}(\boldsymbol{r})=\sigma(\boldsymbol{r}) \boldsymbol{E}(\boldsymbol{r})$. All other currents are considered primary $\boldsymbol{j}^{p}(\boldsymbol{r})=\boldsymbol{j}(\boldsymbol{r})-\boldsymbol{j}^{\boldsymbol{v}}(\boldsymbol{r})$, which, as described by Tripp [49], can be considered to be the sum of the impressed neural current and the microscopic passive cellular currents. The division of the current as primary $\boldsymbol{j}^{p}(\boldsymbol{r})$ and passive $\boldsymbol{j}^{\boldsymbol{v}}(\boldsymbol{r})$ is to emphasize that neural activity in a region gives rise to macroscopic primary currents in that same region that may then flow passively throughout the rest of the conducting medium.

Because of the quasi-static assumptions, the electric field can be modeled as the gradient of a scalar potential, $\boldsymbol{E}(\boldsymbol{r})=$ $-\nabla v(\boldsymbol{r})$. Substituting our interpretation of $\boldsymbol{j}(\boldsymbol{r})$ into (1) yields

$$
\boldsymbol{b}(\boldsymbol{r})=\frac{\mu_{0}}{4 \pi} \int_{G}\left(\boldsymbol{j}^{p}\left(\boldsymbol{r}^{\prime}\right)-\sigma\left(\boldsymbol{r}^{\prime}\right) \nabla v\left(\boldsymbol{r}^{\prime}\right)\right) \times \boldsymbol{d} / d^{3} d \boldsymbol{r}^{\prime}
$$

The typical head model assumes that the head may be represented by three to five regions, e.g., scalp, skull, cerebrospinal fluid, gray matter, and white matter, and that the conductivity $\sigma(\boldsymbol{r})$ is constant and isotropic within these regions. The gradient of the conductivity is, therefore, zero except at the surfaces between regions, which allows the volume integrals to be reworked into surface integrals. We assume our volume can be divided into $M+1$ regions with conductivities $\sigma_{i}, i=$ $1, \cdots, M+1$, which includes the nonconducting region outside of the head. These regions are separated by a total of $m \geq M$ surfaces $S_{i}$. Through simple vector identities, we can rewrite the volume integral in (2) as a sum of surface integrals ([19], cf. [27], [45], [49])

$$
\begin{aligned}
\boldsymbol{b}(\boldsymbol{r})= & \boldsymbol{b}_{\infty}(\boldsymbol{r})-\frac{\mu_{0}}{4 \pi} \sum_{i=1}^{m}\left(\sigma_{i}^{-}-\sigma_{i}^{+}\right) \\
& \cdot\left(\int_{S_{i}} v\left(\boldsymbol{r}^{\prime}\right) \boldsymbol{n}_{i}\left(\boldsymbol{r}^{\prime}\right) \times \boldsymbol{d} / d^{3}\right) d \boldsymbol{r}^{\prime}
\end{aligned}
$$

where $\boldsymbol{n}_{i}(\boldsymbol{r})$ is the "outward" directed unit vector normal to the $i$ th surface, and the "+" ("-") superscript indicates the conductivity outside (inside) the $i$ th surface. The primary field $\boldsymbol{b}_{\infty}(\boldsymbol{r})$ is

$$
\boldsymbol{b}_{\infty}(\boldsymbol{r})=\frac{\mu_{0}}{4 \pi} \int_{G} \boldsymbol{j}^{p}\left(\boldsymbol{r}^{\prime}\right) \times \boldsymbol{d} / d^{3} d \boldsymbol{r}^{\prime}
$$

which is the magnetic field observed at $\boldsymbol{r}$ due to the primary current only. If no boundaries were present, then $\boldsymbol{b}_{\infty}(\boldsymbol{r})$ would represent the magnetic field generated by a primary source in an infinite homogeneous medium.

To compute the magnetic field using (3) we must first know the potential $v(\boldsymbol{r})$ on all boundaries. Using Green's theorem, we can obtain a surface integral equation for $v(\boldsymbol{r})$ (see [2], [17], [18], and [45] for details)

$$
\begin{aligned}
\sigma_{0} v_{\infty}(\boldsymbol{r})= & \frac{\left(\sigma_{j}^{-}+\sigma_{j}^{+}\right)}{2} v(\boldsymbol{r})+\frac{1}{4 \pi} \sum_{i=1}^{m}\left(\sigma_{i}^{-}-\sigma_{i}^{+}\right) \\
& \cdot \int_{S_{i}} v\left(\boldsymbol{r}^{\prime}\right) \boldsymbol{n}_{i}\left(\boldsymbol{r}^{\prime}\right) \cdot \boldsymbol{d} / d^{3} d \boldsymbol{r}^{\prime}, \quad \boldsymbol{r} \in S_{j}
\end{aligned}
$$

where we have assumed all surfaces are smooth, and $v_{\infty}(\boldsymbol{r})$ is the primary potential, i.e., the solution for the infinite 
homogeneous medium of unit conductivity $\sigma_{0}$ due to the primary current $\boldsymbol{j}^{p}(\boldsymbol{r})$

$$
v_{\infty}(\boldsymbol{r})=\frac{1}{4 \pi \sigma_{0}} \int_{G} \boldsymbol{j}^{p}\left(\boldsymbol{r}^{\prime}\right) \cdot \boldsymbol{d} / d^{3} d \boldsymbol{r}^{\prime}
$$

Equations (3) and (5), therefore, form our general set of boundary integral equations for solving the forward problem for scalp potentials (EEG) and external magnetic fields (MEG). If we assume that the primary current exists only at a discrete point, i.e., the primary current source is a current dipole with moment $\boldsymbol{q}$ located at $\boldsymbol{r}_{q}$, then $\boldsymbol{b}_{\infty}(\boldsymbol{r})$ and $v_{\infty}(\boldsymbol{r})$ can be simplified as

$$
\begin{aligned}
& \boldsymbol{b}_{\infty}(\boldsymbol{r})=\left(\mu_{0} / 4 \pi\right) \boldsymbol{q} \times \boldsymbol{d} / d^{3} \\
& v_{\infty}(\boldsymbol{r})=\left(1 / 4 \pi \sigma_{0}\right) \boldsymbol{q} \cdot \boldsymbol{d} / d^{3} .
\end{aligned}
$$

The key modeling assumptions are that the fields are quasistatic and that the shape of the homogeneous regions of the head are known and of known constant isotropic conductivity. Refinements of the models for anisotropic conductivities may be found in [38], [44], and [52] and references therein, but we will restrict our attention to the isotropic case.

\section{Solutions FOR Spherical HeAd Models}

For the case where the head is assumed to comprise a set of nested concentric spheres, each of constant conductivity, analytic solutions exist for both MEG (cf. [45]) and EEG (cf. [6]). Analytic solutions for other head shapes have been presented, such as prolate and oblate spheroids [11] or eccentric spheres [8], and numerical solutions for narrow or wide ellipsoids are presented in [9]. In these presentations of other head shapes, dipole localization errors are presented for the simplifying case of spherical models, and the conclusions are that the deviations between spherical models and these other smooth shapes did not appear to greatly affect source localization. We will, therefore, focus this review on the spherical solutions only.

\section{A. MEG, Spherically Symmetric Conductor}

In general, to solve the forward MEG problem (3), we must first solve (5) for the surface potential $v(\boldsymbol{r})$ on all surfaces. This, therefore, also solves the EEG forward problem. An important exception to this "two-step" process is in the case of the concentric spherical head model, where the MEG forward problem can be solved directly. The radial component of the field at sensor location $\boldsymbol{r}$ is computed as $b_{r}(\boldsymbol{r})=\boldsymbol{b}(\boldsymbol{r}) \cdot \boldsymbol{r} / r$, and for a spherically symmetric conductor, the vector normal to the surface is easily expressed as $\boldsymbol{n}\left(\boldsymbol{r}^{\prime}\right)=\boldsymbol{r}^{\prime} / \boldsymbol{r}^{\prime}$ for all $\boldsymbol{r}^{\prime}$ on all surfaces. In this case, the contribution of the passive currents to $b_{r}(\boldsymbol{r})$ vanishes, since substitution and expansion of (3) yields $\boldsymbol{r} \cdot \boldsymbol{r}^{\prime} \times \boldsymbol{d}=0$. Thus $b_{r}(\boldsymbol{r})$ is simply calculated from the well-known primary current model

$$
\begin{aligned}
b_{r}(\boldsymbol{r}) & =b_{\infty}(\boldsymbol{r}) \cdot \boldsymbol{r} / r=\left(\mu_{0} / 4 \pi\right)\left(\boldsymbol{q} \times \boldsymbol{d} / d^{3} \cdot \boldsymbol{r} / r\right) \\
& =\left(\mu_{0} / 4 \pi\right) \boldsymbol{r} \times \boldsymbol{r}_{q} \cdot \boldsymbol{q} /\left(r d^{3}\right)
\end{aligned}
$$

This formula shows that a radially oriented MEG sensor sees only the dipole moment and not the volume currents.

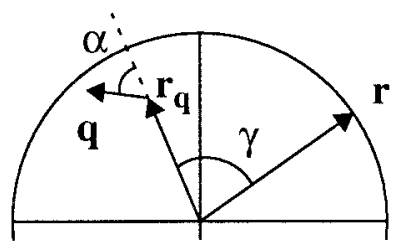

Fig. 1 From [52], the angle between vectors pointing to surface position $\mathbf{r}$ and dipole location $\mathbf{r}_{\mathbf{q}}$ is denoted $\gamma$. The angle the dipole $\mathbf{q}$ makes with the radial direction at $\mathbf{r}_{\mathbf{q}}$ is denoted $\alpha$. The angle between the plane formed by $\mathbf{r}_{\mathbf{q}}$ and $\mathbf{q}$, and the plane formed by $\mathbf{r}_{\mathbf{q}}$ and $\mathbf{r}$ is denoted $\beta$.

If the sensors are not radially oriented, then the effects of the volume currents must be included; however, as shown by Ilmoniemi et al. [29] and Sarvas [45], the full magnetic field for nonradially oriented sensors outside a set of concentric spheres may still be calculated without explicit consideration of the volume currents. Since no currents exist outside the head, both approaches use the radial magnetic field $b_{r}(\boldsymbol{r})$ to derive the scalar magnetic potential $u(\boldsymbol{r})$. The full magnetic field is then derived as the gradient of this scalar. Sarvas' formula $\boldsymbol{b}(\boldsymbol{r})=-\mu_{0} \nabla u(\boldsymbol{r})$ for outside the spherical conductor in Cartesian coordinates is ([45], cf. [27] (34), and [28])

$$
\boldsymbol{b}(\boldsymbol{r})=\frac{\mu_{0}}{4 \pi F^{2}\left(\boldsymbol{r}, \boldsymbol{r}_{q}\right)}\left(F\left(\boldsymbol{r}, \boldsymbol{r}_{q}\right) \boldsymbol{q} \times \boldsymbol{r}_{q}-\left(\boldsymbol{q} \times \boldsymbol{r}_{q} \cdot \boldsymbol{r} \nabla F\left(\boldsymbol{r}, \boldsymbol{r}_{q}\right)\right)\right.
$$

where the scalar function $F\left(\boldsymbol{r}, \boldsymbol{r}_{q}\right)$ and the vector function $\nabla F\left(\boldsymbol{r}, \boldsymbol{r}_{q}\right)$ are

$$
\begin{aligned}
F\left(\boldsymbol{r}, \boldsymbol{r}_{q}\right)= & d\left(r d+r^{2}-\left(\boldsymbol{r}_{q} \cdot \boldsymbol{r}\right)\right) \\
\nabla F\left(\boldsymbol{r}, \boldsymbol{r}_{q}\right)= & \left(\frac{d^{2}}{r}+\frac{(\boldsymbol{d} \cdot \boldsymbol{r})}{d}+2 d+2 r\right) \boldsymbol{r} \\
& -\left(d+2 r+\frac{(\boldsymbol{d} \cdot \boldsymbol{r})}{d}\right) \boldsymbol{r}_{q} .
\end{aligned}
$$

\section{B. EEG, Spherically Symmetric Conductor}

The simplest case in EEG is a single spherical shell head model, i.e., the entire conducting volume is modeled as a sphere of constant conductivity $\sigma$. Brody et al. [6] review earlier formulations and present a generalized expression for this single sphere case. Similarly, Rush and Driscoll [44] review some of the early solutions to single and homogeneous spheres, then present solutions for both anisotropic and multisphere models. Here, we give the form of the solution as recently presented by Zhang in [52], with reference to the geometry in Fig. 1. The signed dipole intensity can be represented by its radial and tangential components, $q_{r}=q \cos \alpha$ and $q_{t}=q \sin \alpha$. The potential can then be expressed as the sum of two potentials, $v^{1}\left(\boldsymbol{r} ; \boldsymbol{r}_{q}, \boldsymbol{q}\right)=v_{r}^{1}\left(\boldsymbol{r} ; \boldsymbol{r}_{q}, \boldsymbol{q}\right)+v_{t}^{1}\left(\boldsymbol{r} ; \boldsymbol{r}_{q}, \boldsymbol{q}\right)$, where

$$
\begin{aligned}
& v_{r}^{1}\left(\boldsymbol{r} ; \boldsymbol{r}_{q}, \boldsymbol{q}\right) \\
& \quad=\left(\frac{q_{r}}{4 \pi \sigma}\right)\left(\frac{2\left(r \cos \gamma-r_{q}\right)}{d^{3}}+\frac{1}{r_{q} d}-\frac{1}{r r_{q}}\right) \\
& v_{t}^{1}\left(\boldsymbol{r} ; \boldsymbol{r}_{q}, \boldsymbol{q}\right) \\
& \quad=\left(\frac{q_{t}}{4 \pi \sigma}\right) \cos \beta \sin \gamma\left(\frac{2 r}{d^{3}}+\frac{d+r}{r d\left(r-r_{q} \cos \gamma+d\right)}\right) .
\end{aligned}
$$


Our explicit statement of the dependence of the potential on $\left(\boldsymbol{r}_{q}, \boldsymbol{q}\right)$ will be made clear below.

The single spherical shell is too unrealistic as a model for the head due to the large difference between the conductivities of brain and skull. The typical multishell spherical model includes three layers for the brain, skull, and scalp; some also include a cerebrospinal fluid layer. The multishell case of $M$ spherical shells requires the evaluation of an infinite series. The infinite series presentation by Zhang in [52] is especially compact compared to earlier presentations (cf. [44])

$$
\begin{aligned}
v^{M}\left(\boldsymbol{r} ; \boldsymbol{r}_{q}, \boldsymbol{q}\right)= & \frac{q}{4 \pi \sigma_{M} r^{2}} \sum_{n=1}^{\infty} \frac{2 n+1}{n}\left(\frac{r_{q}}{r}\right)^{n-1} \\
& \cdot f_{n}\left(n \cos \alpha P_{n}(\cos \gamma)+\cos \beta \sin \alpha\right. \\
& \left.\cdot P_{n}^{1}(\cos \gamma)\right)
\end{aligned}
$$

where $P_{n}$ and $P_{n}^{1}$ are the Legendre and associated Legendre polynomials, respectively, and

$$
f_{n}=\frac{n}{n m_{22}+(1+n) m_{21}} \text {. }
$$

The coefficients $m_{22}$ and $m_{21}$ are found from (17), shown at the bottom of the page, where the conductivities are arranged from the innermost sphere to the outer most, $\sigma_{1}, \cdots, \sigma_{M}$, corresponding to the radii of the spheres $r_{1}<r_{2}<\cdots<r_{m}$, and the matrices in (17) are noncommuting with the highest index matrix applied first. See [52] for details. Similarly, see [38], in which the infinite series analytic solution to the multilayer isotropic model is presented in Cartesian coordinates and the dipole moment clearly separated.

When computing the solution to this forward problem, the infinite series in (15) must be truncated or approximated. Various approximations for the multishell case have been proposed [1], [3], [38], [52]. In [38], de Munck and Peters consider the more general case of anisotropic conductivities, and the infinite series is substituted with one which converges more rapidly. Recent empirical work on closed-form approximations by Berg and Scherg [3], and related theoretical studies by Zhang [52], describe a valid method for approximating the infinite series with as few as three evaluations of scaled forms of the single shell model (13) and (14). For a given $M$-shell head model, these so-called "Berg parameters" [52] can be designated as $\left\{\lambda_{1}, \mu_{1}, \lambda_{2}, \mu_{2}, \lambda_{3}, \mu_{3}\right\}$ (see [3] and [52] for definitions). The potential in the $M$-shell is then approximated as

$$
\begin{aligned}
v^{M}\left(\boldsymbol{r} ; \boldsymbol{r}_{q}, q\right) \cong & v^{1}\left(\boldsymbol{r} ; \mu_{1} \boldsymbol{r}_{q}, \lambda_{1} \boldsymbol{q}\right)+v^{1}\left(\boldsymbol{r} ; \mu_{2} \boldsymbol{r}_{q}, \lambda_{2} \boldsymbol{q}\right) \\
& +v^{1}\left(\boldsymbol{r} ; \mu_{3} \boldsymbol{r}_{q}, \lambda_{3} \boldsymbol{q}\right)
\end{aligned}
$$

This method uses the true dipole location $\boldsymbol{r}_{q}$ to select three dipole locations along the same radial line and uses these dipoles to evaluate the single shell model (13) and (14) three times. These three evaluations are then scaled and summed. The scalar values are a predetermined function of conductivities and shell thicknesses, and evaluation of the $M$ shell model becomes quite fast and accurate. See [3] and [52] for examples and details on computing these "Berg parameters." These approximations are enhancements to an earlier approximation presented in [1] (the "Airy correction factor"), as well as extensions of the approximation theory presented by de Munck in [38].

\section{Matrix Kernels for Spherical Heads}

If the primary sources were completely specified in both location and moment, then implementation of the above formulas could proceed directly. The inverse problem, however, involves finding a suitable set of primary sources that adequately describe the data recorded by a limited set of sensors. As we showed in [33], the inverse problem can often be better approached if we separate the linear moment parameters $\boldsymbol{q}$ from the nonlinear location parameters $\boldsymbol{r}_{\boldsymbol{q}}$. The inverse problem can then be approached as an explicit function of just the location parameters, reducing the complexity of the solution search.

In this section we factor the solutions from Section II-B as the product of a "field kernel" and the dipole moment. We will represent each model solution as the MEG vector $\boldsymbol{b}(\boldsymbol{r})=\boldsymbol{K}\left(\boldsymbol{r}, \boldsymbol{r}_{\boldsymbol{q}}\right) \boldsymbol{q}$ or the EEG scalar $v(\boldsymbol{r})=\boldsymbol{k}^{T}\left(\boldsymbol{r}, \boldsymbol{r}_{\boldsymbol{q}}\right) \boldsymbol{q}$, where $\boldsymbol{K}\left(\boldsymbol{r}, \boldsymbol{r}_{\boldsymbol{q}}\right)$ is a $3 \times 3$ matrix kernel and $\boldsymbol{k}\left(\boldsymbol{r}, \boldsymbol{r}_{\boldsymbol{q}}\right)$ is a $3 \times 1$ vector kernel. These field kernels are then combined with the sensor characteristics to yield discrete matrices of lead fields [6], [49] that are clearly separated from the dipole moments.

Before presenting the table of field kernels, we mention several properties useful in reducing the solutions to kernel forms. We first note the triple scalar product identity $\boldsymbol{a} \times \boldsymbol{b} \cdot \boldsymbol{c}=$ $\boldsymbol{a} \cdot \boldsymbol{b} \times \boldsymbol{c}$, and the anticommutative property of the cross-product, $\boldsymbol{a} \times \boldsymbol{b}=-\boldsymbol{b} \times \boldsymbol{a}$, both of which are useful in the reduction of the formulas. To simplify the algebraic manipulation of the cross-product, we convert the operation to the product of a matrix and a vector and explicitly state all vectors in their Cartesian forms

$$
\boldsymbol{a} \times \boldsymbol{b}=C_{\boldsymbol{a}} \boldsymbol{b}=\left[\begin{array}{ccc}
0 & -a_{z} & a_{y} \\
a_{z} & 0 & -a_{x} \\
-a_{y} & a_{x} & 0
\end{array}\right]\left[\begin{array}{l}
b_{x} \\
b_{y} \\
b_{z}
\end{array}\right] .
$$

These identities and substitutions are useful in reducing the Sarvas formula (10) to the product of a matrix kernel and the dipole moment.

For the EEG solutions, we prefer to avoid calculations involving explicit transcendental functions, which are computationally expensive. In (13), we note that $q_{r}=q \cos \alpha=$ $q\left(\boldsymbol{r}_{q} \cdot \boldsymbol{q}\right) /\left(r_{q} q\right)$. With similar conversions for the other transcendental functions, we note that $q \sin \alpha \cos \beta \sin \gamma$ may be equivalently expressed as $(\boldsymbol{r} \cdot \boldsymbol{q}) / r-\cos \gamma\left(\left(\boldsymbol{r}_{\boldsymbol{q}} \cdot \boldsymbol{q}\right) / r_{q}\right)$. Using

$$
\left[\begin{array}{ll}
m_{11} & m_{12} \\
m_{21} & m_{22}
\end{array}\right]=\frac{1}{(2 n+1)^{M-1}} \prod_{k=1}^{M-1}\left[\begin{array}{cc}
n+\frac{(n+1) \sigma_{k}}{\sigma_{k+1}} & (n+1)\left(\frac{\sigma_{k}}{\sigma_{k+1}}-1\right)\left(\frac{r_{q}}{r_{k}}\right)^{2 n+1} \\
n\left(\frac{\sigma_{k}}{\sigma_{k+1}}-1\right)\left(\frac{r_{k}}{r_{q}}\right)^{2 n+1} & (n+1)+\frac{n \sigma_{k}}{\sigma_{k+1}}
\end{array}\right]
$$


these substitutions in (13) and (14) yields

$$
\begin{aligned}
v_{r}^{1}(\boldsymbol{r})= & \left.\frac{q\left(\boldsymbol{r}_{q} \cdot \boldsymbol{q}\right) /\left(r_{q} q\right)}{4 \pi \sigma}\right)\left(\frac{2\left(r\left(\left(\boldsymbol{r} \cdot \boldsymbol{r}_{q}\right) /\left(r r_{q}\right)\right)-r_{q}\right)}{d^{3}}\right. \\
& \left.\quad+\frac{1}{r_{q} d}-\frac{1}{r r_{q}}\right) \\
= & c_{1} \boldsymbol{r}_{q} \cdot \boldsymbol{q}
\end{aligned}
$$

and

$$
\begin{aligned}
v_{t}^{1}(\boldsymbol{r})= & \left(\frac{q \sin \alpha}{4 \pi \sigma}\right) \cos \beta \sin \gamma \\
& \cdot\left(\frac{2 r}{d^{3}}+\frac{d+r}{r d\left(r-r_{q}\left(\left(\boldsymbol{r} \cdot \boldsymbol{r}_{q}\right) /\left(r r_{q}\right)\right)+d\right)}\right) \\
= & \frac{1}{4 \pi \sigma}\left(\frac{(\boldsymbol{r} \cdot \boldsymbol{q})}{r}-\left(\frac{\left(\boldsymbol{r} \cdot \boldsymbol{r}_{q}\right)}{r r_{q}}\right)\left(\frac{\left(\boldsymbol{r}_{\boldsymbol{q}} \cdot \boldsymbol{q}\right)}{r_{q}}\right)\right) \\
& \cdot\left(\frac{2 r}{d^{3}}+\frac{d+r}{d\left(r^{2}-\left(\boldsymbol{r} \cdot \boldsymbol{r}_{q}\right)+d r\right)}\right) \\
= & \left(r_{q}^{2}(\boldsymbol{r} \cdot \boldsymbol{q})-\left(\boldsymbol{r} \cdot \boldsymbol{r}_{q}\right)\left(\boldsymbol{r}_{\boldsymbol{q}} \cdot \boldsymbol{q}\right)\right) c_{2}
\end{aligned}
$$

where the scalar coefficients $c_{1}$ and $c_{2}$ are defined as

$$
\begin{aligned}
& c_{1} \equiv \frac{1}{4 \pi \sigma r_{q}^{2}}\left(2 \frac{\left(\boldsymbol{d} \cdot \boldsymbol{r}_{q}\right)}{d^{3}}+\frac{1}{d}-\frac{1}{r}\right) \\
& c_{2} \equiv \frac{1}{4 \pi \sigma r_{q}^{2}}\left(\frac{2}{d^{3}}+\frac{d+r}{r F\left(\boldsymbol{r}, \boldsymbol{r}_{q}\right)}\right) .
\end{aligned}
$$

Thus, the single shell EEG model solution can be expressed as

$$
v^{1}(\boldsymbol{r})=\left(\left(c_{1}-c_{2}\left(\boldsymbol{r} \cdot \boldsymbol{r}_{q}\right)\right) \boldsymbol{r}_{q}+c_{2} r_{q}^{2} \boldsymbol{r}\right) \cdot \boldsymbol{q}
$$

This novel form of the EEG single-sphere solution is an algebraic reformulation of the original presentation in [6], but with an emphasis on vector notation. Our form includes the term $F\left(\boldsymbol{r}, \boldsymbol{r}_{q}\right)$, which also appears in the MEG solution (11). This reworking of the single shell EEG solution now has a clear separation between the vector kernel and the dipole moment, and all calculations are in vector Cartesian coordinates. Additionally, our simplification to the coefficients in (25) and (26) highlights the computational similarities between the Sarvas formula in (10) and this single-shell formula. For the multishell formula, similar reductions may be applied to the infinite series in (15), as presented by de Munck and Peters [38]. Our preference is to use the Berg parameters in (18) to effect the approximation to the multishell model using a single shell equivalent. Using these properties and observations, the E/MEG solutions presented in the previous section can be reduced to their kernel forms as listed in Table I.

\section{BOUNDARY Element Methods}

Clearly, the head is not spherical, and improvements in the forward calculations can be effected by replacing the spherical geometry with a more realistic head shape extracted from anatomical images. Since it is not currently possible to obtain accurate estimates of spatially varying tissue conductivities, the head is typically assumed to consist of a set of contiguous isotropic regions, each of constant conductivity, yielding the boundary integral equations of Section II. Equation (5) is a Fredholm integral of the second kind for the unknown surface potential $v(\boldsymbol{r})$, and must be solved numerically for realistic head geometries. Boundary element methods (BEM's) for solving (5) have been widely studied in the MEG and EEG literature (cf. [4], [9], [10], [15], [16], [26], [27], [30]-[32], [35]-[37], [39], [40], [43], [46], [47], and [50]). Here, we review the BEM approach to solving the E/MEG forward problem using the method of weighted residuals as a framework. We then describe how most of the previously published methods in this field are of the "constant collocation," "linear collocation," or "constant Galerkin" forms. We also present our own novel investigations of "linear Galerkin" weighting, as well as the effects of the "isolated skull approach" [26] on MEG and EEG solutions.

\section{A. Method of Weighted Residuals}

We can express the right-hand side of (5) as a linear operator acting on the potential function $v(\boldsymbol{r})$, i.e., $L(v(\boldsymbol{r}))=v_{\infty}(\boldsymbol{r})$. In the forward problem, the source and hence the function $v_{\infty}(\boldsymbol{r})$ is known, and the task is to determine $v(\boldsymbol{r})$, such that the residual $L(v(\boldsymbol{r}))-v_{\infty}(\boldsymbol{r})$ is as small as possible. The standard method of weighted residuals solves this problem using a weighting function $w(\boldsymbol{r})$, i.e., we solve the related problem

$$
\int\left(L\left(v\left(\boldsymbol{r}^{\prime}\right)\right)-v_{\infty}\left(\boldsymbol{r}^{\prime}\right)\right) w\left(\boldsymbol{r}^{\prime}\right) d \boldsymbol{r}^{\prime}=0
$$

or equivalently

$$
\left(w(\boldsymbol{r}), v_{\infty}(\boldsymbol{r})\right)=(w(\boldsymbol{r}), L(v(\boldsymbol{r})))
$$

where $(\cdot, \cdot)$ denotes the inner product of the two functions. The integration is over the domain of the unknown potential function $v(\boldsymbol{r})$, which in (5) is restricted to the two-dimensional surfaces.

The selection of a particular weighting function determines the class of error method. The BEM restricts the weighting functions to a finite combination of $N$ known linearly independent basis functions $\psi_{n}(\boldsymbol{r})$

$$
w(\boldsymbol{r})=\sum_{n=1}^{N} \beta_{n} \psi_{n}(\boldsymbol{r}) .
$$

The coefficients $\beta_{n}$ are arbitrary, such that $w(\boldsymbol{r})$ spans this $N$ dimensional space. Therefore, (29) must hold for each of the individual basis functions $\psi_{n}(\boldsymbol{r})$, yielding $N$ equations

$$
\left(\psi_{i}(\boldsymbol{r}), v_{\infty}(\boldsymbol{r})\right)=\left(\psi_{i}(\boldsymbol{r}), L(v(\boldsymbol{r}))\right), \quad i=1,2, \cdots, N
$$

We next need to transform the unknown potential function $v(\boldsymbol{r})$ into something more tractable for numerical computing. The BEM approximates $v(\boldsymbol{r})$ as another finite combination of $N$ known linearly independent basis functions $\varphi_{n}(\boldsymbol{r})$

$$
v(\boldsymbol{r}) \cong \sum_{n=1}^{N} v_{n} \varphi_{n}(\boldsymbol{r}) .
$$

The unknown coefficients $v_{n}$ are the nodal parameters, which are functions of the nodes or nodal points $\boldsymbol{r}_{\boldsymbol{n}}$. The basis function $\varphi_{n}$ is chosen with the property that $\varphi_{n}\left(\boldsymbol{r}_{\boldsymbol{n}}\right)=1$ at the 
TABLE I

The E/MEg Models for Different Head Models Are Presented in the Left Column, with the Corresponding Equation from the Text Indicated in Parentheses. The Solution Kernels Are Given Either in a Matrix Form $\boldsymbol{K}\left(\boldsymbol{r}, \boldsymbol{r}_{\boldsymbol{q}}\right)$ or a Vector Form $\boldsymbol{k}\left(\boldsymbol{r}, \boldsymbol{r}_{\boldsymbol{q}}\right)$, ANd the Subscripts on the Kernels Relate to the SPeCific E/MEG Model. The Kernels Can Be Applied as

$\boldsymbol{b}(\boldsymbol{r})=\boldsymbol{K}\left(\boldsymbol{r}, \boldsymbol{r}_{\boldsymbol{q}}\right) \boldsymbol{q}$ OR $\boldsymbol{v}(\boldsymbol{r})=\boldsymbol{k}\left(\boldsymbol{r}, \boldsymbol{r}_{\boldsymbol{q}}\right)^{T} \boldsymbol{q}$, WhERE $\boldsymbol{r}_{\boldsymbol{q}}$ AND $\boldsymbol{q}$ ARE THE LOCATION AND MOMENT OF AN EQUivalent CURRENT Dipole

\begin{tabular}{|c|c|}
\hline Model & Solution Kernel \\
\hline $\begin{array}{c}\text { EEG, Infinite Homogeneous Model (8) } \\
\text { (Primary Potential) }\end{array}$ & $\begin{aligned} \boldsymbol{k}_{\infty}\left(\boldsymbol{r}, \boldsymbol{r}_{q}\right) & =\left[\frac{1}{4 \pi} \frac{\boldsymbol{d}}{d^{3}}\right] \\
\text { where } \boldsymbol{d} & =\boldsymbol{r}-\boldsymbol{r}_{q}\end{aligned}$ \\
\hline $\begin{array}{c}\text { MEG, Infinite Homogeneous Model (7) } \\
\text { (Primary Field) }\end{array}$ & $\begin{array}{l}\boldsymbol{K}_{\infty}\left(\boldsymbol{r}, \boldsymbol{r}_{q}\right)=\left[\frac{\mu_{0}}{4 \pi} \frac{C_{\boldsymbol{r}_{q}}-C_{\boldsymbol{r}}}{d^{3}}\right] \\
\left.\text { where } C_{(}\right) \text {is defined in (19). }\end{array}$ \\
\hline $\begin{array}{l}\text { MEG Spherical, Radial Field (9) } \\
\qquad\left(b_{r}(\boldsymbol{r})=\boldsymbol{r} \cdot \boldsymbol{b}_{\infty}(\boldsymbol{r}) / \boldsymbol{r}\right)\end{array}$ & $\boldsymbol{k}_{r}\left(\boldsymbol{r}, \boldsymbol{r}_{q}\right)=\left[\frac{\mu_{0}}{4 \pi} \frac{C_{r} \boldsymbol{r}_{q}}{r d^{3}}\right]$ \\
\hline $\begin{array}{l}\text { MEG Spherical, Full Field (10) } \\
\text { (Sarvas Model) }\end{array}$ & $\begin{array}{l}\qquad \boldsymbol{K}_{s}\left(\boldsymbol{r}, \boldsymbol{r}_{q}\right)=\left[\frac{\mu_{0}}{4 \pi} \frac{\left[\nabla F \boldsymbol{r}^{T}-F \boldsymbol{I}\right]}{F^{2}} \boldsymbol{C}_{\boldsymbol{r}_{q}}\right] \\
\text { where } F \text { and } \nabla F \text { are defined in (11) and (12). }\end{array}$ \\
\hline EEG Spherical, Single Shell (13), (14) & $\begin{array}{l}\boldsymbol{k}_{1}\left(\boldsymbol{r}, \boldsymbol{r}_{q}\right)=\left[\left(c_{1}-c_{2}\left(\boldsymbol{r} \cdot \boldsymbol{r}_{q}\right)\right) \boldsymbol{r}_{q}+c_{2} r_{q}^{2} \boldsymbol{r}\right] \\
\text { where } c_{1} \text { and } c_{2} \text { are defined in (25) and (26). }\end{array}$ \\
\hline EEG Spherical, $M$ Shell, Approximate (18) & $\begin{array}{l}\boldsymbol{k}_{3}\left(\boldsymbol{r}, \boldsymbol{r}_{q}\right) \cong \\
\lambda_{1} \boldsymbol{k}_{1}\left(\boldsymbol{r}, \mu_{1} \boldsymbol{r}_{q}\right)+\lambda_{2} \boldsymbol{k}_{1}\left(\boldsymbol{r}, \mu_{2} \boldsymbol{r}_{q}\right)+\lambda_{3} \boldsymbol{k}_{1}\left(\boldsymbol{r}, \mu_{3} \boldsymbol{r}_{q}\right) \\
\quad \text { where }\left\{\mu_{i}, \lambda_{i}\right\} \text { are "Berg Parameters"[3], [52]. }\end{array}$ \\
\hline EEG BEM (41) & $\begin{array}{c}\boldsymbol{k}_{\text {bem }}\left(\boldsymbol{r}, \boldsymbol{r}_{q}\right)=\left[\left[\varphi_{1}(\boldsymbol{r}), \ldots, \varphi_{N}(\boldsymbol{r})\right] \tilde{\boldsymbol{H}}^{-1} \boldsymbol{G}_{\infty}\right] \\
\text { where } \tilde{\boldsymbol{H}} \text { is "deflated" from (33), and } \\
\boldsymbol{G}_{\infty} \text { is defined by (40). }\end{array}$ \\
\hline MEG BEM (42) & $\begin{array}{c}\boldsymbol{K}_{\text {bem }}\left(\boldsymbol{r}, \boldsymbol{r}_{q}\right)=\left[\boldsymbol{K}_{\infty}\left(\boldsymbol{r}, \boldsymbol{r}_{q}\right)+\boldsymbol{A}(\boldsymbol{r}) \tilde{\boldsymbol{H}}^{-1} \boldsymbol{G}_{\infty}\right] \\
\text { where } \boldsymbol{A}(\boldsymbol{r}) \text { is from [15] or [37]. }\end{array}$ \\
\hline
\end{tabular}

nodal point and is otherwise zero at all other nodes. This choice of nodal points and basis functions yields the equivalence in (32) between a nodal parameter and nodal point as $v_{n}=v\left(\boldsymbol{r}_{\boldsymbol{n}}\right)$. Substituting (32) into (31) yields a system of equations

$$
\begin{aligned}
{\left[\begin{array}{c}
\left(\psi_{1}(\boldsymbol{r}), v_{\infty}(\boldsymbol{r})\right) \\
\cdots \\
\left(\psi_{N}(\boldsymbol{r}), v_{\infty}(\boldsymbol{r})\right)
\end{array}\right] } & \\
= & {\left[\begin{array}{ccc}
\left(\psi_{1}(\boldsymbol{r}), L\left(\varphi_{1}(\boldsymbol{r})\right)\right. & \cdots & \left(\psi_{1}(\boldsymbol{r}), L\left(\varphi_{N}(\boldsymbol{r})\right)\right) \\
\cdots & \cdots & \cdots \\
\left(\psi_{N}(\boldsymbol{r}), L\left(\varphi_{1}(\boldsymbol{r})\right)\right) & \cdots & \left(\psi_{N}(\boldsymbol{r}), L\left(\varphi_{N}(\boldsymbol{r})\right)\right)
\end{array}\right] } \\
& \cdot\left[\begin{array}{l}
v_{1} \\
\cdots \\
v_{N}
\end{array}\right] .
\end{aligned}
$$

Thus (33) represents the BEM in its more general form. By design, the only unknown is the vector of $N$ coefficients, $\left\{v_{i}\right\}$. Once the coefficients have been calculated, then $v(\boldsymbol{r})$ can be computed on any surface using (32). Selection of the potential and weighting basis functions determines not only the adequacy of the approximation in (32), but also the complexity of inner product calculations in (33).

The two most common weighting functions in the E/MEG literature are the collocation and Galerkin forms. In the collocation form, $\psi_{i}(\boldsymbol{r})$ is chosen as the Dirac delta function $\delta\left(\boldsymbol{r}-\boldsymbol{r}_{i}\right)$, where $\boldsymbol{r}_{i}$ is the corresponding nodal point. In other words, the weighted residual equation (28) is satisfied at certain collocation points instead of in an average sense, 
such that (33) becomes

$$
\left[\begin{array}{c}
v_{\infty}\left(\boldsymbol{r}_{1}\right) \\
\cdots \\
v_{\infty}\left(\boldsymbol{r}_{N}\right)
\end{array}\right]=\left[\begin{array}{ccc}
L\left(\varphi_{1}\left(\boldsymbol{r}_{1}\right)\right) & \cdots & L\left(\varphi_{N}\left(\boldsymbol{r}_{1}\right)\right) \\
\cdots & \cdots & \cdots \\
L\left(\varphi_{1}\left(\boldsymbol{r}_{N}\right)\right) & \cdots & L\left(\varphi_{N}\left(\boldsymbol{r}_{N}\right)\right)
\end{array}\right]\left[\begin{array}{c}
v_{1} \\
\cdots \\
v_{N}
\end{array}\right] .
$$

The obvious advantage of (34) is that the inner products in (33) reduce to simpler function evaluations at the nodal points. The Galerkin form is a weighted residual method for which the weighting basis functions are identical to the potential basis functions, i.e., $\psi_{i}(\boldsymbol{r})=\varphi_{i}(\boldsymbol{r})$.

The two most common potential basis functions used in E/MEG are constant and linear. Each closed surface is first tessellated into $T$ planar triangles with $T / 2+2$ vertices. The constant potential basis function assumes that $\varphi_{n}^{c}(\boldsymbol{r})=1$ for $\boldsymbol{r}$ on the $n$th triangle. The resulting potential $v(\boldsymbol{r})$ is discontinuous on the boundary between two triangles and at vertices. The nodal point for each potential basis function is usually assumed to lie at the centroid of the triangle, resulting in $N=T$ basis functions.

For the linear potential basis function, let $\boldsymbol{r}_{i}, \boldsymbol{r}_{j}$, and $\boldsymbol{r}_{k}$ be three vertices of the $n$th triangle ordered in such a way that the permutation $\boldsymbol{r}_{i} \rightarrow \boldsymbol{r}_{j} \rightarrow \boldsymbol{r}_{k}$ corresponds by the right-hand rule to the outward normal of the surface. The linear basis functions are then defined as (cf. [37])

$$
\begin{aligned}
\varphi_{i}^{l}(\boldsymbol{r}) & =\frac{\boldsymbol{r} \cdot\left(\boldsymbol{r}_{j} \times \boldsymbol{r}_{k}\right)}{\boldsymbol{r}_{i} \cdot\left(\boldsymbol{r}_{j} \times \boldsymbol{r}_{k}\right)}, \quad \varphi_{j}^{l}(\boldsymbol{r})=\frac{\boldsymbol{r} \cdot\left(\boldsymbol{r}_{k} \times \boldsymbol{r}_{i}\right)}{\boldsymbol{r}_{i} \cdot\left(\boldsymbol{r}_{j} \times \boldsymbol{r}_{k}\right)}, \\
\varphi_{k}^{l}(\boldsymbol{r}) & =\frac{\boldsymbol{r} \cdot\left(\boldsymbol{r}_{i} \times \boldsymbol{r}_{j}\right)}{\boldsymbol{r}_{i} \cdot\left(\boldsymbol{r}_{j} \times \boldsymbol{r}_{k}\right)}
\end{aligned}
$$

where $\boldsymbol{r}$ is in the interior of the triangle. Thus any point in the interior of a triangle is represented by three basis functions, any point along a border between two triangles is represented by two basis functions, and the points at the vertices are the nodal points, represented by a single basis. For $T$ triangles on a closed surface, the linear approximation has $N=T / 2+2$ basis functions.

These weighting and potential basis functions can be substituted in the $N \times N$ system of equations in (33), which we can represent as

$$
g=H v
$$

where $\boldsymbol{g}$ is an $N \times 1$ vector, $\boldsymbol{H}$ is an $N \times N$ matrix, and $\boldsymbol{v}$ is the $N \times 1$ vector of unknown coefficients. The matrix $\boldsymbol{H}$ is a function of the known basis functions $\psi(\boldsymbol{r})$ and $\varphi(\boldsymbol{r})$ and the head geometry. The "geometry matrix" can be precomputed without knowledge of the primary currents or sensor locations. Depending on the choice of weighting and potential basis functions, the inner products in $\boldsymbol{H}$ may be computed analytically or by using a numerical integration scheme.

In E/MEG, the Neumann boundary condition used to generate (5) leads to a well-known ambiguity; an arbitrary constant potential may be added to any valid solution. The result is a singularity in the matrix $\boldsymbol{H}$, but the eigenvector associated with the zero eigenvalue is simply the constant vector (all elements equal). The matrix can be "deflated" [7], [31] to yield a generally well-conditioned matrix $\tilde{\boldsymbol{H}}$ that is directly invertible. With this deflation, the vector of unknown basis coefficients is

$$
\boldsymbol{v}=\tilde{\boldsymbol{H}}^{-1} \boldsymbol{g} .
$$

The potentials are then found from (32) for an arbitrary point $\boldsymbol{r}$ on a surface using

$$
v(\boldsymbol{r}) \cong\left[\varphi_{1}(\boldsymbol{r}), \cdots, \varphi_{N}(\boldsymbol{r})\right] \boldsymbol{v}=\left[\varphi_{1}(\boldsymbol{r}), \cdots, \varphi_{N}(\boldsymbol{r})\right] \tilde{\boldsymbol{H}}^{-1} \boldsymbol{g}
$$

As discussed in Hämäläinen and Sarvas [26] and Meijs et al. [32], numerical implementations for multilayer models may yield unacceptable errors in voltage potentials at the scalp surface. They introduce an approximation they refer to as the isolated skull approach (ISA) in which the skull is modeled as perfectly insulating; the result of the resulting field calculations are then mapped back into the multishell model. Although presented in [26] as a "two-pass" algorithm (first calculate the one shell model, then the updated multishell model), the effects of their approximation are readily folded into a single modified matrix $\boldsymbol{H}$. Srebro [47] presents a recent modification of this concept to yield an alternative "one-pass" algorithm.

\section{B. Matrix Kernels}

To reduce the BEM equations to the inner product of a kernel and the dipole moment, each element in the vector $\boldsymbol{g}$ in (38) can be represented as

$$
g_{i}=\left(\psi_{i}(\boldsymbol{r}), v_{\infty}(\boldsymbol{r})\right)=\left(\psi_{i}(\boldsymbol{r}), \boldsymbol{k}_{\infty}\left(\boldsymbol{r}, \boldsymbol{r}_{q}\right)\right)^{T} \boldsymbol{q}
$$

where the specific form will be dependent on the choice of the weighting function $\psi(\boldsymbol{r})$, and $\boldsymbol{k}_{\infty}\left(\boldsymbol{r}, \boldsymbol{r}_{\boldsymbol{q}}\right)$ is defined in Table I. The dipole moment can, therefore, be separated from the inner product, and for the $N$ basis functions we define an $N \times 3$ matrix $\boldsymbol{G}_{\infty}$ such that

$$
\boldsymbol{g}=\boldsymbol{G}_{\infty} \boldsymbol{q}=\left[\begin{array}{c}
\left(\psi_{1}(\boldsymbol{r}), \boldsymbol{k}_{\infty}\left(\boldsymbol{r}, \boldsymbol{r}_{\boldsymbol{q}}\right)\right)^{T} \\
\cdots \\
\left(\psi_{N}(\boldsymbol{r}), \boldsymbol{k}_{\infty}\left(\boldsymbol{r}, \boldsymbol{r}_{\boldsymbol{q}}\right)\right)^{T}
\end{array}\right] \boldsymbol{q} .
$$

From (32), we see that the potential on any surface is, therefore

$$
\begin{aligned}
v(\boldsymbol{r}) & \cong\left[\varphi_{1}(\boldsymbol{r}), \cdots, \varphi_{N}(\boldsymbol{r})\right] \boldsymbol{v} \\
& =\left[\varphi_{1}(\boldsymbol{r}), \cdots, \varphi_{N}(\boldsymbol{r})\right] \tilde{\boldsymbol{H}}^{-1} \boldsymbol{G}_{\infty} \boldsymbol{q}
\end{aligned}
$$

and the EEG forward problem is solved.

For the MEG solution, we insert (38) into (3). Ferguson et al. [15] and de Munck [37] have shown that the resulting integrations in (3) can be performed exactly for the constant and linear basis functions. The MEG forward problem is, therefore, solved for arbitrary point $\boldsymbol{r}$ as

$$
\boldsymbol{b}(\boldsymbol{r}) \cong \boldsymbol{b}_{\infty}(\boldsymbol{r})+\boldsymbol{A}(\boldsymbol{r}) \boldsymbol{v}
$$

where $\boldsymbol{A}(\boldsymbol{r})$ is a $3 \times N$ matrix found analytically by inserting the basis functions into the integral in (3). We refer the reader to [37, (13)] and [15, (12)] for the explicit calculation of $\boldsymbol{A}(\boldsymbol{r})$, since the definition of terms used becomes quite involved. 


\section{Matrix Inverses and Transfer Matrices}

As noted, the solution of the forward problem is usually incorporated into an inverse method. The inverse problem may require computation of the forward fields for thousands of dipole locations. With the sensor locations known, efficiencies can be realized by precomputing terms independent of the dipole locations. These terms can be combined into transfer matrices that are stored and retrieved at "run-time" for more efficient generation of the gain matrices.

Since the matrix $\tilde{\boldsymbol{H}}$ is independent of both the dipole location and sensor location, it can be precomputed and stored prior to the determination of the sensor locations. In some early work, such as [2], the computational resources were not available to invert $\tilde{\boldsymbol{H}}$ directly, and iterative techniques were instead discussed. While some recent papers continue to use Gauss-Seidel or Jacobi iterations [32], or power series expansions [10], these approaches are, generally, not numerically advisable [20], and the inverse can be more efficiently and stably computed using an LU decomposition as suggested in [25] and [40] and subsequent works. Let the LU decomposition be denoted as $\boldsymbol{L}=\tilde{\boldsymbol{H}}$. The LU decomposition allows efficient and stable calculation of the transfer matrix using Gaussian backsubstitution, and we will emphasize this efficiency by denoting the inverse as $\tilde{H}^{-1}=(\boldsymbol{L} U)^{-1}$.

If we assume a single common reference electrode, the EEG BEM kernel can be concatenated for $m+1$ sensors as

$$
\left[\begin{array}{c}
\varphi_{1}\left(\boldsymbol{r}_{1}\right), \cdots, \varphi_{N}\left(\boldsymbol{r}_{1}\right) \\
\cdots \\
\varphi_{1}\left(\boldsymbol{r}_{m+1}\right), \cdots, \varphi_{N}\left(\boldsymbol{r}_{m+1}\right)
\end{array}\right] \tilde{\boldsymbol{H}}^{-1} \boldsymbol{G}_{\infty}
$$

We can generate the full EEG gain matrix by premultiplying the kernel by the "switching matrix" $W$ which subtracts the $(m+1)$ st sensor from the $m$ single-ended electrode locations [35]. Combining this matrix with (43) allows us to precompute a "transfer matrix" $\boldsymbol{T}_{v}$ that is independent of the dipole location

$$
\left(\boldsymbol{W}\left[\begin{array}{c}
\varphi_{1}\left(\boldsymbol{r}_{1}\right), \cdots, \varphi_{N}\left(\boldsymbol{r}_{1}\right) \\
\cdots \\
\varphi_{1}\left(\boldsymbol{r}_{m+1}\right), \cdots, \varphi_{N}\left(\boldsymbol{r}_{m+1}\right)
\end{array}\right](\boldsymbol{L} \boldsymbol{U})^{-1}\right) \boldsymbol{G}_{\infty}=\boldsymbol{T}_{v} \boldsymbol{G}_{\infty}
$$

where Gaussian backsubstitution is used to efficiently generate $\boldsymbol{T}_{v}$. For $m$ EEG channels and $N$ BEM basis functions this transfer matrix is $m \times N$. The extension to multiple reference electrodes and differential pairs follows easily. At "run-time," one or more candidate dipole locations are selected, the matrix $\boldsymbol{G}_{\infty}$ is formed using (40), then the gain matrix is formed as the product $\boldsymbol{T}_{v} \boldsymbol{G}_{\infty}$. The development of the magnetic field transfer matrix follows similarly.

The precomputation of these EEG and MEG transfer matrices can greatly increase the efficiency of inverse procedures. We note similar descriptions, alternative decompositions [such as the singular value decomposition (SVD)], and the use of such transfer matrices in [4], [16], [25], [39], [40], and [43].

\section{Comparisons of Error Weighting}

The performance of BEM methods is dependent on the selection of the basis and weighting functions. To demonstrate the effects of these weightings, we have applied BEM to a three-shell spherical model, so that numerical comparisons could be made with the known analytic solution. The radii of the model were 88,85 , and $81 \mathrm{~mm}$, and the conductivities were $0.33,0.0042,0.33 \Omega^{-1} \mathrm{~m}^{-1}$ for the scalp, skull, and brain, respectively. The MEG sensors were placed $120 \mathrm{~mm}$ from the center, i.e., $32 \mathrm{~mm}$ from the "scalp" and all oriented in the $x$-direction; the use of nonradial MEG sensor orientations highlights the effect of volume currents on the BEM calculations. The EEG electrodes were assumed to be at the nodal points of the triangles (i.e., centroids for the constant basis and vertices for the linear basis) on the upper hemisphere of the outer most surface.

For the potential basis sets we applied both the constant and linear basis functions, and for the weighting functions we used both collocation and Galerkin methods. Computations were performed with and without the ISA [26]. We used 492 and 1016 nominally equilateral triangles per surface for the constant and linear BEM respectively, so that the degrees of freedom (DOF's) for the linear and constant cases were approximately equal. The average length of the side of triangle on the inner skull was $20.0 \mathrm{~mm}$ in the constant case and $13.9 \mathrm{~mm}$ in the linear case. The matrices for the forward model were "deflated" [7], [31] and the forward gain matrices were computed as described above. We used the approach in [15] to compute the MEG solutions from the BEM calculated potentials.

The dipole was moved along the $z$-axis from $[0,0,3]-[0$, $0,78] \mathrm{mm}$, i.e., to within $3 \mathrm{~mm}$ of the vertex of a triangle tessellated on the inner most shell. The dipole was oriented in each of the three orthogonal directions, $x, y, z$, and the EEG and MEG forward fields calculated for each orientation. The comparison metric used was the relative difference measure (RDM), defined as

$$
\mathrm{RDM}=\sqrt{\left(\boldsymbol{b}_{\mathrm{th}}-\boldsymbol{b}\right)^{T}\left(\boldsymbol{b}_{\mathrm{th}}-\boldsymbol{b}\right) /\left(\boldsymbol{b}_{\mathrm{th}}^{T} \boldsymbol{b}_{\mathrm{th}}\right)}
$$

where $\boldsymbol{b}_{\text {th }}$ and $\boldsymbol{b}$ denote the analytic and numerical EEG or MEG sensor values.

In Fig. 2, the RDM for EEG and MEG are shown for the dipole oriented in the $x$-direction. Since the MEG sensors are also oriented in the $x$-direction, the contribution in (42) from the primary current is completely suppressed, and the RDM reflects the error from calculating the contributions from the volume currents only. For the EEG results without ISA, we observe that "constant Galerkin" (effectively the original Lynn and Timlake [30] approach) and "linear collocation" do not generally improve the RDM over that of the simpler "constant collocation" method. These results are consistent with Schlitt et al. [46] who show errors on the outermost surface using the linear approximation (analogous to our case of linear collocation) that are almost twice as great as when using the constant basis (our constant collocation case). "Linear Galerkin" achieves a better RDM over constant collocation. 


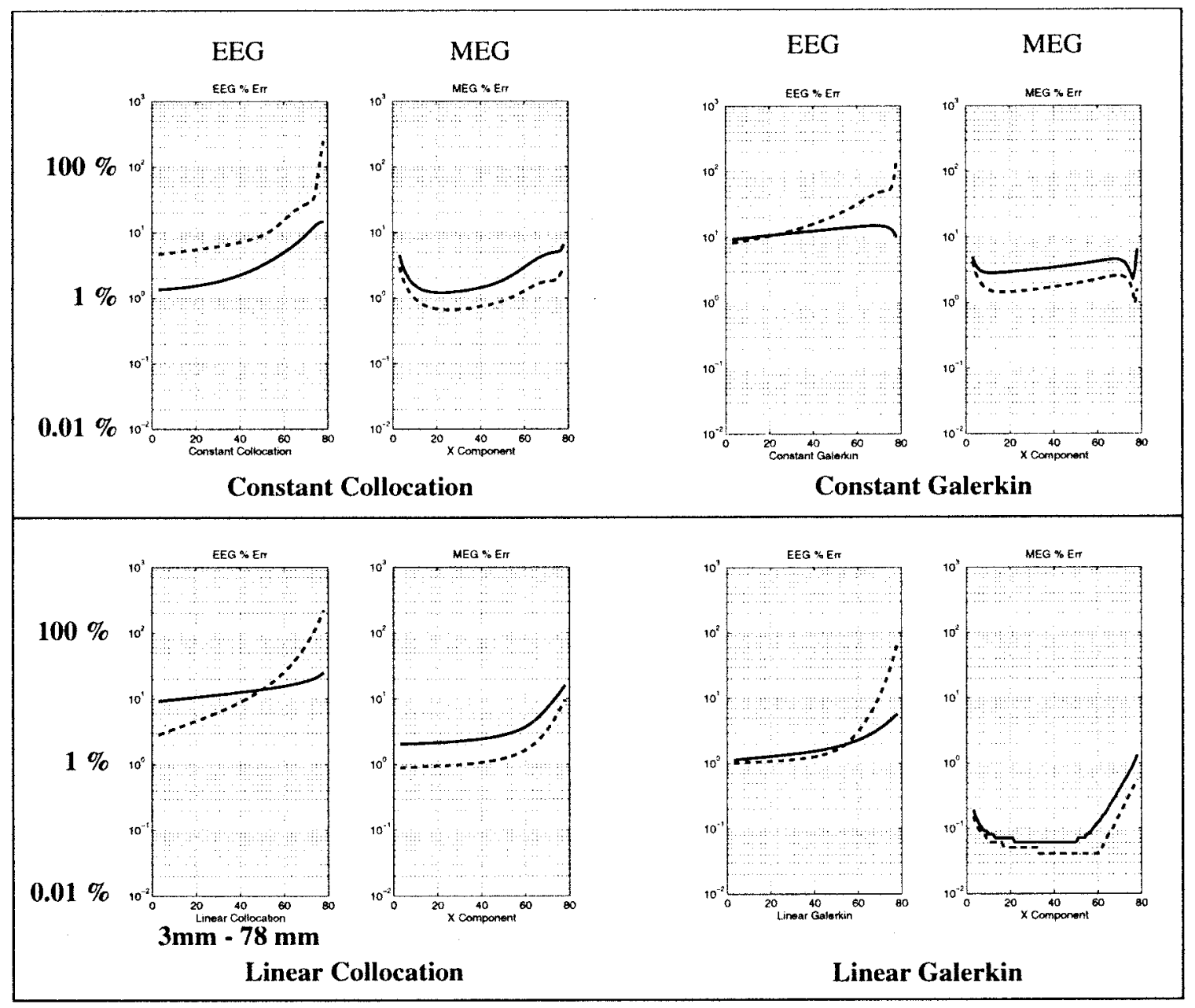

Fig. 2 The RDM (in percent $\log$ scale) versus an $x$-directed dipole. The dipole is positioned along the $z$-axis from $3 \mathrm{~mm}$ to $78 \mathrm{~mm}$, i.e., to within $3 \mathrm{~mm}$ of the radius of the innermost of the three spherical shells; see the text for descriptions of the model and the locations of the EEG and MEG sensors. Since the MEG sensors and dipole are both aligned in the $x$-direction, the MEG sensors measure only the contributions from the volume currents. The top row shows the results using a constant basis function across each triangle; the bottom row assumes a linear basis function. The left two columns use collocation for the weighted residuals, and the right columns use the Galerkin approach, i.e., the error basis is the same as the model basis. The solid line denotes the RDM using the ISA [26], and the dashed line is without ISA. We note that ISA improves the EEG solution for a dipole near the surface, but generally degrades the MEG solution.

In all EEG cases without ISA, the RDM is on the order of $100 \%$ error as the dipole approaches the innermost surface. The EEG results with ISA show a remarkable improvement in the RDM, and we see that both the constant collocation and the linear Galerkin results show about $8 \%$ error as the dipole approaches the inner surface.

In the MEG plots, we observe that in general the RDM is below 10\%, a significant improvement over the EEG RDM. This low RDM confirms the generally held belief that the MEG forward solution is less sensitive to BEM errors. We note that both constant collocation and constant Galerkin are in general better than linear collocation, particularly for dipoles near the surface. We note that ISA does not improve the MEG results, which suggests that although ISA improves the scalp potentials for EEG, the cost is a perturbation of the stronger innermost currents that are detected by MEG. Near the innermost surface, the constant model results show errors of only a few percent, but we see that the linear Galerkin results are dramatically superior to all others, with RDM below $1 \%$ even directly below the surface.
In Fig. 3, we repeat the analysis for a $y$-directed dipole. By rotational symmetry, the EEG results are virtually identical; however, the $x$-directed MEG sensors now measure contributions from both the primary and volume currents. At the sphere center, all dipoles are virtually radially oriented, such that the external MEG should be zero and, therefore, the primary and volume current terms in (42) must now cancel each other. We see that the $y$-directed dipole generates relatively larger RDM than the $x$-directed dipole, for dipoles near the center of the sphere. At shallower depths, both "tangential" directions yield similar RDM's.

In Fig. 4, the dipole is now $z$-directed. Since the dipole was positioned on the $z$-axis, then the $z$-directed dipole represents a radial dipole, for which the external magnetic field is zero and the MEG RDM, therefore, undefined. In this case, we calculated for MEG the root-mean-square (rms) error for a 10-nA-m dipole moment

$$
\operatorname{rms}=\sqrt{\left(\boldsymbol{b}^{T} \boldsymbol{b}\right) / 104}
$$

which gives an indication of the amount of error for a putative dipolar source across the MEG array of 104 sensors. 




Fig. 3 The RDM (in percent $\log$ scale) versus a $Y$-directed dipole along the $z$-axis from $3 \mathrm{~mm}$ to $78 \mathrm{~mm}$. See Fig. 2 for simulation details. Contrasted with Fig. 2, the MEG sensors in this simulation measure contributions from both the primary and volume currents. By symmetry, the EEG results are virtually the same as Fig. 2.

Again, by spherical symmetry, radial dipoles near the center of the head yield EEG RDM's similar to the tangential directions. As this radial dipole approaches the surface, however, the differences in the various approximations become dramatic. At shallow depths, the radial orientation yields a strong potential gradient across the tessellated surface, severely "straining" the constant and linear assumptions. Without the ISA, the EEG RDM's exceed 100\% near the surface, except in the linear Galerkin case. With ISA, the constant Galerkin RDM remains remarkably consistent at all depths, but the linear Galerkin RDM's are again superior, remaining below $6 \%$ even just inside the surface.

For MEG the radial dipoles generate no external magnetic field and the primary and volume currents in (42) should cancel. The rms values in this case reflect the BEM error in canceling the numerically calculated volume current term versus the analytically computed primary current term. As the dipole approaches the innermost surface, we see that the rms error for a 10-nA-m dipole exceeds $100 \mathrm{fT}$, except for the linear Galerkin case. Again, in all cases ISA does not improve the MEG rms. In the linear Galerkin case without ISA, the rms error remains below $6 \mathrm{fT}$, an error level dramatically below all other cases.
These results demonstrate the importance of proper basis selection for both the weighting and potential functions. Since most primary activity is presumed to lie in the cortex, the accuracy of the forward solution for sources within a few millimeters of the inner skull is of vital importance in E/MEG. We have shown that with the number of nodes held constant, differences in RDM error can exceed factors of 100, particularly in the critical region near the inner skull. The ISA in general yields a dramatic improvement for EEG for dipoles near the surface; however, the approximation is somewhat detrimental to MEG calculations.

\section{DISCUSSION}

\section{A. Summary}

In developing the kernels listed in Table I, we were attempting to address aspects of the forward problem in E/MEG that are of particular interest in the development and implementation of inverse methods. One important issue addressed here is the ability to factor out the dipole moment in a matrix formulation of the inverse problem, for both spherical and realistic head geometries. In [33], we showed that the least 


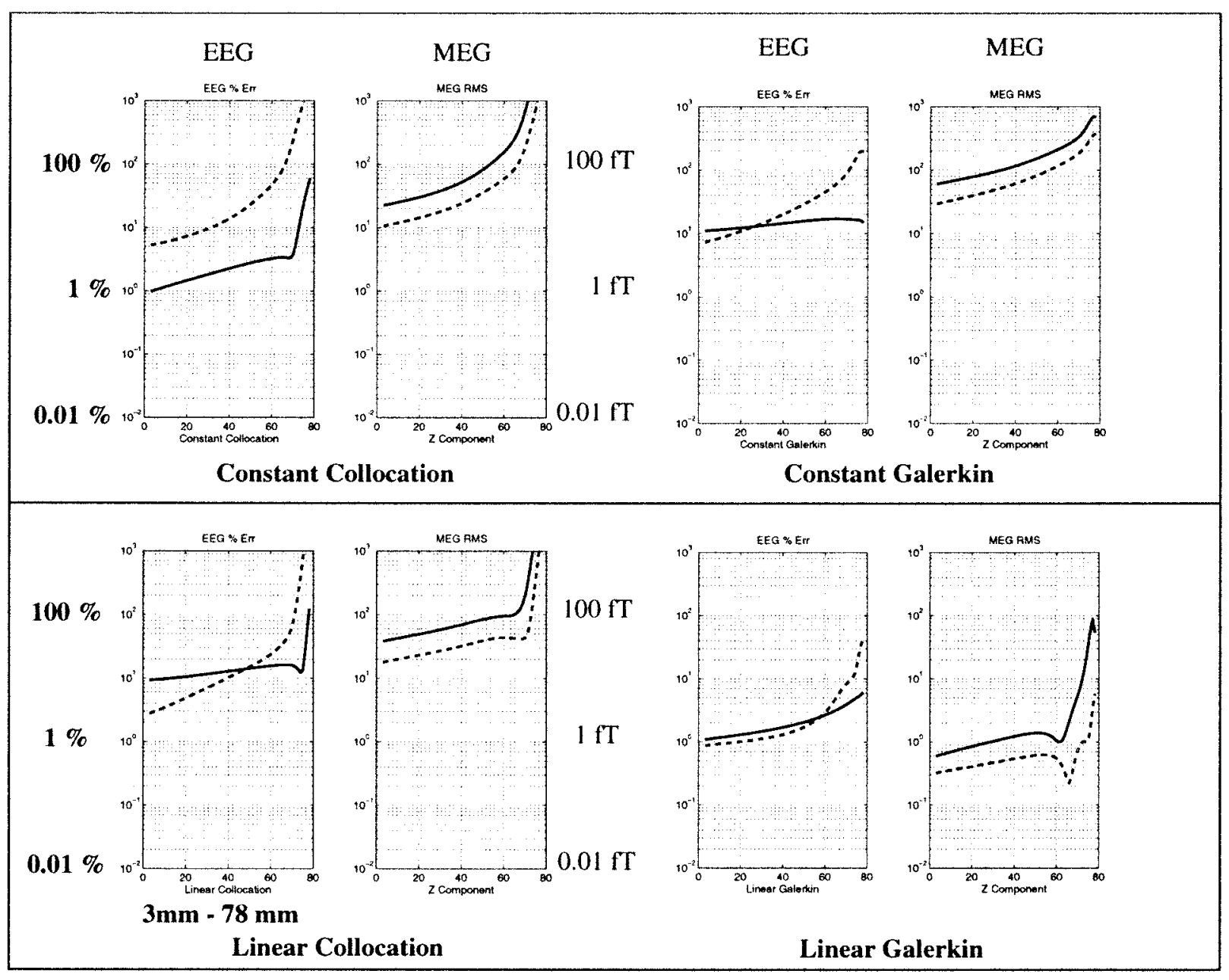

Fig. 4 The RDM (in percent log scale) and rms (in fT log scale) versus a $Z$-directed dipole along the z-axis from $3 \mathrm{~mm}$ to $78 \mathrm{~mm}$. See Fig. 2 for simulation details. Since the dipole is radially oriented in this simulation, the MEG sensors should theoretically measure a zero external field, and the RDM is undefined. In this instance, we plot instead the rms error (in fT) for a $10 \mathrm{nA}-\mathrm{m}$ dipole moment. For example, a $10 \mathrm{nA}-\mathrm{m}$ radial dipole generates about a maximum 6-fT rms error, in the linear Galerkin case without ISA. The radial direction appears to strain the assumptions of constant or linear potentials as the dipole approaches the inner surface, but the linear Galerkin results are dramatically improved over the other techniques.

squares localization problem can be solved efficiently for a complete set of spatio-temporal data by first solving for the linear parameters as a function of the nonlinear ones; this linear solution reduces the problem to a search over the nonlinear parameters only. To do this, we first need to cleanly separate the nonlinear dipole location parameters from the linear moment parameters. Although this separation is theoretically straightforward (cf. [6], [38], and [49]), a unified presentation of the concise forms listed in Table I has not previously been published.

A second goal of our work was to explicitly compare the computational complexity of EEG and MEG forward models. In recent review papers such as [27], [45], and [51], the MEG spherical solutions are nicely developed, but the EEG spherical solutions are omitted, with the possible impression that their formulation is perhaps too complicated to present. When combined with the "Berg" parameters, our reformulations of the single-shell field kernel show computation of the EEG solution to be of the same complexity as the MEG solution.

As the acquisition of anatomical magnetic resonance images as part of an experimental or clinical E/MEG paradigm becomes routine, spherical representations of the head in E/MEG can be replaced with more realistic geometries. In general, these geometries require numerical solutions, and our development and presentation show EEG and MEG BEM kernels to be of similar complexity. Both modalities require the specification of the conductivities and boundaries in generating the final transfer matrix. Finally, we note that when using realistic geometries, numerical solution of the forward problem involves several design parameters. The numerical results in the previous section highlight the dramatic effects that these parameters can have. In an attempt to explain these effects, we discuss next an illustrative example. We follow this with a review of the existing E/MEG BEM literature in terms of the development presented in Section IV.

\section{B. Weighted Residuals}

A simple illustrative example will serve to explain the effects that different error weightings can produce in E/MEG forward solutions. Let $f(x)=x^{2}$ be a quadratic function to be approximated by a constant or a linear basis function, 


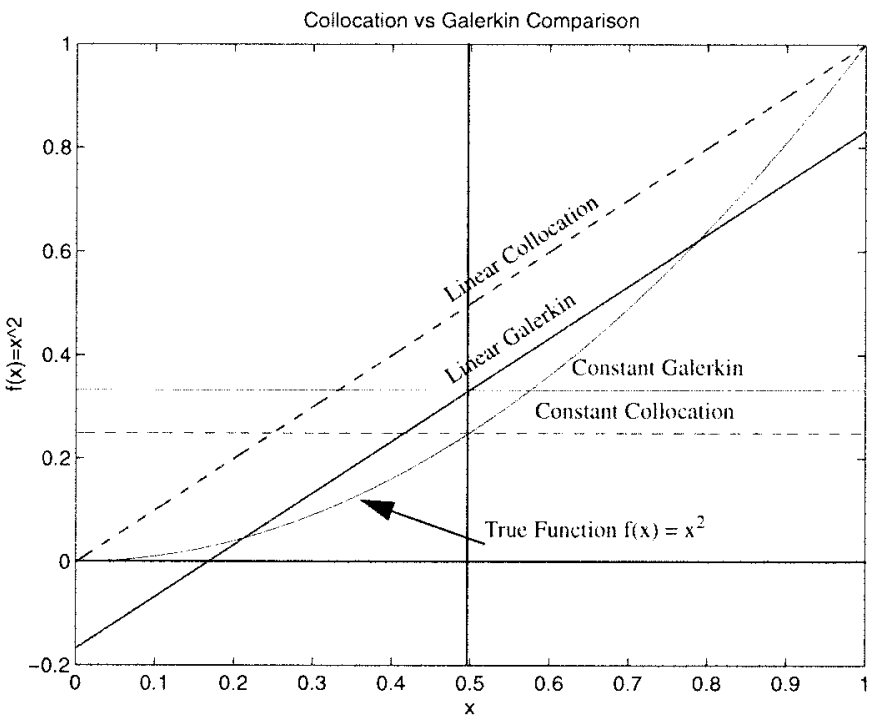

Fig. 5 Simple example suggested by S. Ferguson (private communication February 1996) to show the effects of approximation and error weighting. The true function over the interval is $f(x)=x^{2}$, and its true integral over the interval is $1 / 3$. The collocation forms precisely match the true function at their respective nodal points, $x=0.5$, and $x=0,1$. The linear collocation has twice the integration error over that of constant collocation for this interval The Galerkin forms are in error at the nodal points, but both forms precisely integrate to the correct value, $1 / 3$. Thus collocation may be seen as minimizing the error at specific points, while Galerkin weighting minimizes the error in a more global sense.

as illustrated in Fig. 5. The weighted residual expression is, therefore, $\int\left(f(x)-x^{2}\right) w(x) d x$, which we will evaluate on the interval $[0,1]$. For constant collocation, we approximate $f(x)$ as a constant function, $\varphi_{0}(x)=1$, with a nodal point at the midpoint, $x=0.5$. Substitution into the weighted residual expression and minimization yields $v_{0}=1 / 4$. Similarly, for linear collocation, the two basis functions are $\varphi_{0}(x)=1-x$ and $\varphi_{1}(x)=x$ with corresponding nodal points $x=0$ and $x=1$. Minimizing this weighted residual yields $v_{0}=0$ and $v_{1}=1$.

Both the constant and linear approximations to $f(x)$ are overlaid in Fig. 5, and we see that both collocation error methods yield the correct values of $f(x)$ at their nodal points. Next we consider Galerkin weightings over the same interval

$$
\int_{0}^{1}\left(\sum v_{i} \varphi_{i}(x)-x^{2}\right) \varphi_{j}(x) d x
$$

yielding $v_{0}=1 / 3$ for constant Galerkin, and $v_{0}=-1 / 6, v_{1}=$ $5 / 6$ for linear Galerkin. These solutions are overlaid on Fig. 5.

Two features distinguish the Galerkin solutions from the collocation methods: i) the Galerkin approximations to $f(x)$ have larger errors at the nodal points than the collocation results (which are perfect at the nodal points in this example); and ii) both Galerkin approximations integrate to the same value as the true function over this interval, $\int f(x) d x=1 / 3$. By comparison, the constant and linear collocation forms integrate to $1 / 4$ and $1 / 2$, respectively, where we note that the linear collocation error is twice the constant collocation error.

The differences in integration error versus nodal evaluation error in this example has implications for EEG and MEG. In EEG, we are primarily interested in the evaluation of the potential at discrete sensor sites about the scalp. Thus the collocation approach implies we might actually achieve better error performance than Galerkin if we assign nodal points to the sensor sites. In MEG, however, the sensor sites are a function of the integral of the potential over all surfaces, and the Galerkin approach implies that we might achieve better error control over the continuum of potentials and hence better MEG approximations.

This example helps explain some of the differences we note among linear collocation and both forms of constant bases in our simulations; however, we found linear Galerkin to be generally superior. For the same number of DOF's, the triangles can be smaller in the linear case than in the constant case, and we are weighting the error over the entire triangle, not just the nodal points. These differences are most notable near the inner skull surface; we contrast these with the much larger relative errors in the same region in [16], [47] (most other BEM publications do not include error results for sources so close to the inner skull).

\section{Other BEM Approaches}

The weighted residual approach in Section IV is not the only numerical approach to solving an integral equation. This approach, however, is a useful interpretation for much of the work on BEM's in E/MEG forward problems, although the terminology "collocation" and "Galerkin" as presented here and in [36] is not often used. One of the first papers often cited for the computational solution of the E/MEG integral equations is Lynn and Timlake [30], which presented a formal error analysis for the case of the average error across planar triangles, over which the potential had been assumed constant. Lynn and Timlake noted that the work of [2] and others were ad hoc special cases of their rigorous method, which we have referred to as constant Galerkin, since their average error is the same as the method of weighted residuals with constant basis sets for both the weighting and potential functions.

In many other papers, the "geometry" matrix in the Galerkin form of (33) is approximated by the values at the triangular centroids (cf. [27]), which some authors also refer to as the "discretization points" (cf. [32] and [37]). Many of these forms may be more formally described as constant collocation, since the weighting function is the Dirac delta. Often, however, authors begin with the constant Galerkin form of Lynn and Timlake, then shift to a collocation form for the geometry matrix, yielding (probably) a hybrid mix between collocation and Galerkin.

Another hybrid based on the constant assumption is that of Meijs et al. [32]. They assume a constant potential across each triangle, but their discretization points (or nodal points) are at the triangle vertices (rather than centroids), where the potential is generally discontinuous. In Schlitt et al. [46], their "vertex method constant" potential follows the constant hybrid method in [32], and their center of gravity "COG" technique is equivalent to our constant collocation.

Other work has focused on assuming a linear variation across each triangle. In [37], de Munck presents a linear basis for the potential across each triangle, with the equations 
assessed at discrete points, yielding our equivalent of linear collocation BEM. He presents analytic solutions for some of the central integrations in the linear collocation approach for EEG. Schlitt et al. [46] compare a linear collocation BEM to the two constant BEM's discussed above; analytic solutions to some of the integrations are also included. In [15], Ferguson et al. present analytic solutions for both constant and linear assumptions for the integrals in the MEG forward model (3), formally completing the model for these basis functions. More recently, Ferguson and Stroink [14] discuss several variations of constant and linear approximations and their impact on forward model accuracy.

In solving for (5), two boundary constraints were used: the potential and the currents normal to this surface must be continuous across the boundary. As detailed in [2], [17], [18], and [45], these constraints were used analytically to yield (5), which is a function of the unknown potentials only. In [5], Brebbia et al. refer to this approach as an "indirect" BEM, since the potential function (5) is first analytically derived, before applying the BEM. The "direct" method sets up a system of equations based on both the potentials and their normal derivatives, then proceeds to solve numerically for the unknowns. Examples of the "direct" method in E/MEG are Boemmel et al. [4], Urankar [50], and Fletcher et al. [16], who apply collocation BEM to these "direct" equations. Boemmel et al. [4] and Urankar [50] have also presented analytic solutions for these "direct" kernels using a linear basis function. Fletcher et al. [16] present comparisons of their direct technique with other BEM forms. See [5] for a more complete discussion on "direct" versus "indirect" methods.

In Gonzalez et al. [21], a collocation technique is described wherein the number of collocation points may exceed the number of potential basis functions, and the potential basis functions themselves are drawn from a Fourier description of the surfaces rather than planar triangles. Hafner [23] refers to this overspecification of collocation points as "generalized point matching," and multipolar expansions of surface boundary parameterizations are discussed in the framework of a "generalized multipole technique" or "multiple multipoles" [23]. Approaches of this type have not been widely studied in the E/MEG literature (but, see also [24]).

From this brief review of BEM as applied to E/MEG, we observe many variations and hybrids, yet few of these publications place their methods into a common framework or standard BEM terminology, such as used in [5] and [48]. We hope that the framework presented here illustrates the important issues of "constant" versus "linear" (with some presentation of "quadratic" in [15], [16], and [37]) potential basis function, and "collocation" versus "Galerkin" weighting basis functions. We note also that these issues are also discussed in the more general computational electromagnetics community - see [13] and [22] for example.

Even with the numerical technique and bases selected, each of the elements in the geometry matrix $H$ in (36) generally still requires an integration or multiple integrations. The works in [4], [15], [37], [41], [46], and [50] present analytic solutions to many of these integrals. We note, however, that Strang and Fix [48, p. 98] caution that accurate analytic integration of the matrix elements does not necessarily lead to better results when compared to numerical integration of these elements: "We regret to report that these inexact numerical integrations have even been shown in some cases to improve the quality of the solutions. This is one instance ... in which computational experiments yield results which are frustrating to the mathematical analyst but nevertheless numerically valid and important."

\section{CONCLUSIONS}

We have shown that the forward problem in MEG and EEG can be expressed in a matrix formulation in which the various components of the model are factored. This common framework includes MEG and EEG data, spherical and realistic head geometries, sensor orientation, gradiometer and differential measurement effects, and constrained and unconstrained dipole orientations. A key component of this factorization is the field kernel that relates a dipole with arbitrary orientation and location to the surface potentials and the (vector) magnetic field outside the head for spherical and realistic head geometries. These field kernels are summarized in Table I.

In the case of MEG, the lead field can be specified as separate matrices for the field kernel, sensor orientations and gradiometer configurations. For methods using constrained dipole orientations, the dipole moments are explicitly factored, such that their orientations are easily incorporated in the gain matrix. Similarly for EEG we can separate the field kernels and switching matrices, as well as reduce the "run-time" computations for inverse techniques using precomputation of source independent terms and, in the case of BEM methods, through calculating the surface potentials at the sensor locations only.

Using the recent theoretical work in approximating the infinite series for the EEG spherical calculation, we have shown that the computational complexities of EEG and MEG are approximately equal for both spherical and BEM models. Through the use of our gain matrix framework, we can easily compare different modeling assumptions using a common inverse method, or conversely, compare different inverse methods using a common forward model. Combining the two modalities into a single gain matrix is relatively simple using the formulation presented here, although scaling differences in the data and noise must be accounted for to effectively use this combined E/MEG matrix in an inverse procedure.

Although the spherical head model may not be sufficiently accurate, we have presented numerical results demonstrating that BEM methods can also produce large errors. Consequently, details of specific BEM implementations are necessary when they are used as part of an inverse procedure, in order to delineate the effects of numerical errors in the forward solution on the inverse method. More sophisticated head models employing anisotropic conductivities will need to address these same numerical issues in their BEM or finite element method (FEM) solutions to the forward problem.

\section{ACKNOWLEDGMENT}

The authors would like to thank J. Chang and T. Zhang of the Signal and Image Processing Institute at the University 
of Southern California, Los Angeles, for their assistance in manipulating the transcendental functions in Section III and programming the results of Section V. They would also like to thank Dr. S. Ferguson for suggesting the simple example in Fig. 5, which helps illustrate some of the basis and error weighting issues. Finally, they also thank the anonymous reviewers for their helpful guidance in the revision.

\section{REFERENCES}

[1] J. P. Ary, S. A. Klein, and D. H. Fender, "Location of sources of evoked scalp potentials: Corrections for skull and scalp thickness," IEEE Trans. Biomed. Eng., vol. BME-28, pp. 447-452, June 1981

[2] R. C. Barr, T. C. Pilkington, J. P. Boineau, M. S. Spach, "Determining surface potentials from current dipoles, with application to electrocardiography,” IEEE Trans. Biomed. Eng., Apr. 1966, pp. 88-92.

[3] P. Berg, and M. Scherg, "A fast method for forward computation of multiple-shell spherical head models," Electroenceph. Clin. Neurophysiol., vol. 90, pp. 58-64, 1994.

[4] F. R. Boemmel, R. Roeckelein, and L. Urankar, "Boundary element solution of biomagnetic problems," IEEE Trans. Magn., vol. 29, pp. 1395-1398, Mar. 1993

[5] C. A. Brebbia, J. C. F. Telles, and L. C. Wrobel, Boundary Element Techniques. Berlin, Germany: Springer-Verlag, 1984.

[6] D. A. Brody, F. H. Terry, and R. E. Ideker, "Eccentric dipole in a spherical medium: Generalized expression for surface potentials," IEEE Trans. Biomed. Eng., vol. BME-20, Mar. 1973, pp. 141-143.

[7] T. F. Chan, "Deflated decomposition solutions of nearly singular systems," SIAM J. Numer. Anal., vol. 21, pp. 738-754, 1984.

[8] B. N. Cuffin, "Eccentric spheres models of the head," IEEE Trans. Biomed. Eng., vol. 38, pp. 871-878, Sept. 1991.

[9] __ "Effects of head shapes on EEG's and MEG's," IEEE Trans. Biomed. Eng., vol. 37, pp. 15-22, Nov. 1990.

[10] "A method for localizing EEG sources in realistic head models," IEEE Trans. Biomed. Eng., vol. 42, pp. 68-71, 1995.

[11] B. N. Cuffin and D. Cohen, "Magnetic fields of a dipole in special volume conductor shapes," IEEE Trans. Biomed. Eng., vol. BME-24, pp. 372-381, July 1977

[12] A. M. Dale and M. I. Sereno, "Improved localization of cortical activity by combining EEG and MEG with MRI cortical surface reconstruction: A linear approach," J. Cogn. Neurosci., vol. 5, no. 2, pp. 162-176, 1993.

[13] D. G. Dudley, "Comments on "Variational nature of Galerkin and non-Galerkin moment method solutions," IEEE Trans. Antennas and Propagat., vol. 45, pp. 1062-1063, June 1997.

[14] A. S. Ferguson and G. Stroink, "Factors affecting the accuracy of the boundary element method in the forward problem. I. Calculating the surface potentials," IEEE Trans. Biomed. Eng., vol. 44, pp. 1139-1155, Nov. 1997.

[15] A. S. Ferguson, X. Zhang, and G. Stroink, "A complete linear discretization for calculating the magnetic field using the boundary element method," IEEE Trans. Biomed. Eng., vol. 41, pp. 455-459, 1994.

[16] D. J. Fletcher, A. Anir, D. L. Jewett, and G. Fein, "Improved method for computation of potentials in a realistic head shape model," IEEE Trans. Biomed. Eng., vol. 11, pp. 1094-1104, 1995.

[17] D. B. Geselowitz, "Green's theorem and potentials in a volume conductor," IEEE Trans. Biomed. Eng., vol. BME-14, pp. 54-55, Jan. 1967.

[18] _ "On bioelectric potentials in an inhomogeneous volume conductor," Biophys. J., vol. 7, pp. 1-11, 1967.

[19] _ _ "On the magnetic field generated outside an inhomogeneous volume conductor by internal volume currents," IEEE Trans. Magn., vol. BME-6, pp. 346-347, 1970.

[20] G. H. Golub and C. F. Van Loan, Matrix Computations, 2nd ed. Baltimore, MD: Johns Hopkins Univ. Press, 1984.

[21] S. Gonzalez, R. Grave de Peralta, R. Biscay, J. C. Jimenez, R. D. Pascual, J. Lemagne, and P. A. Valdes, "Projective methods for the magnetic direct problem," Advances in Biomagnetism, S. J. Williamson, M. Hoke, G. Stroink, and M. Kotani, Eds. New York: Plenum, 1989, pp. $615-618$

[22] R. D. Graglia, R. J. Luebbers, and D. R. Wilton, "Special issue on advanced numerical techniques in electromagnetics," IEEE Trans. Antennas and Propagat., vol 45, pp. 313-315, Mar. 1997.

[23] C. Hafner, The Generalized Multipole Technique for Computational Electromagnetics. Boston, MA: Artech House, 1990.

[24] J. Haueisen, C. Hafner, H. Nowak, and H. Brauer, "Neuromagnetic field computation using the multiple multipole method," Int. J. Numer. Modeling, vol. 9, nos. 1-2, pp. 145-158, 1996.
[25] M. S. Hämäläinen, “A 24-channel planar gradiometer: System design and analysis of neuromagnetic data," Advances in Biomagnetism, S. J. Williamson, M. Hoke, G. Stroink, and M. Kotani, Eds. New York: Plenum, 1989, pp. 639-644.

[26] M. S. Hämäläinen and J. Sarvas, "Realistic conductor geometry model of the human head for interpretation of neuromagnetic data," IEEE Trans. Biomed. Eng., vol. 36, pp. 165-171, 1989.

[27] M. Hämäläinen, R. Hari, R. J. Ilmoniemi, J. Knuutila, and O. V. Lounasmaa, "Magnetoencephalography-Theory, instrumentation, and applications to noninvasive studies of the working human brain," Rev. Modern Phys., vol. 65, no. 2, pp. 413-497, 1993.

[28] L. Heller and D. B. van Hulsteyn, "Brain stimulation using electromagnetic sources: theoretical aspects," Biophys. J., vol. 63, pp. 129-138, July 1992.

[29] R. J. Ilmoniemi, M. S. Hamalainen, and J. Knuutila, "The forward and inverse problems in the spherical model," in Biomagnetism: Applications and Theory, H. Weinberg, G. Stroink, and T. Katila, Eds. New York: Pergamon, 1985 , pp. 278-282.

[30] M. S. Lynn and W. P. Timlake, "The numerical solution of the singular integral equations of potential theory," Numerische Mathematik, vol 11, pp. 77-98, 1968

[31] _ , "The use of multiple deflations in the numerical solution of singular systems of equations with applications to potential theory," SIAM J. Numer. Analysis, vol. 5, no. 2, pp. 303-322, 1968

[32] J. W. H. Meijs, O. W. Weier, M. J. Peters, and A. van Oosterom, "On the numerical accuracy of the boundary element method," IEEE Trans. Biomed. Eng., vol. 36, pp. 1038-1049, 1989.

[33] J. Mosher, P. Lewis, and R. Leahy, "Multiple dipole modeling and localization from spatio-temporal MEG data," IEEE Trans. Biomed. Eng., vol. 39, pp. 541-557, 1992.

[34] J. C. Mosher, M. E. Spencer, R. M. Leahy, and P. S. Lewis, "Error bounds for EEG and MEG dipole source localization," Electroenceph. and Clin. Neurophys., vol. 86, pp. 303-321, June 1993.

[35] J. C. Mosher, R. M. Leahy, and P. S. Lewis, "Matrix kernels for MEG and EEG source localization and imaging," in Proc. IEEE Acoustics, Speech, and Signal Processing Conf. 1995, vol. 5, Detroit, MI, May 7-12, 1995, pp. 2943-2946.

[36] J. C. Mosher, C. M. Chang, and R. M. Leahy, "Comparison of the constant and linear boundary element method for EEG and MEG forward modeling," in Biomag96: Advances in Biomagnetism Research, C. J. Aine, E. R. Flynn, Y. Okada, G. Stroink, S. J. Swithenby, and C. C. Wood, Eds. New York: Springer-Verlag, 1998, to be published.

[37] J. C. de Munck, "A linear discretization of the volume conductor integral equations using analytically integrated elements," IEEE Trans. Biomed. Eng., vol. 39, pp. 986-990, 1992.

[38] J. C. deMunck and M. J. Peters, "A fast method to compute the potential in the multisphere model," IEEE Trans. Biomed. Eng., vol. 40, pp. 1166-1174, Nov. 1993.

[39] J. Nenonen, C. J. Purcell, B. M. Horacek, G. Stroink, and T. Katila, "Magnetocardiographic functional localization using a current dipole in a realistic torso," IEEE Trans. Biomed. Eng., vol. 38, pp. 658-664, 1991.

[40] T. F. Oostendorp and A. van Oosterom, "Source parameter estimation in inhomogenous volume conductors of arbitrary shape," IEEE Trans. Biomed. Eng., vol 36, pp. 382-391, Mar. 1989.

[41] A. van Oosterom and J. Strakee, "The solid angle of a plane triangle," IEEE Trans. Biomed. Eng., vol. BME-30, pp. 125-126, 1983.

[42] J. W. Phillips, R. M. Leahy, and J. C. Mosher, "MEG-based imaging of focal neuronal current sources," IEEE Trans. Med. Imag., vol. 16, pp. 338-348, June 1997.

[43] C. J. Purcell and G. Stroink, "Moving dipole inverse solutions using realistic torso models," IEEE Trans. Biomed. Eng., vol. 38, pp. 82-84, Jan. 1991.

[44] S. Rush and D. A. Driscoll, "EEG electrode sensitivity-An application of reciprocity," IEEE Trans. Biomed. Eng., vol. BME-16, pp. 15-22, Jan. 1969

[45] J. Sarvas, "Basic mathematical and electromagnetic concepts of the biomagnetic inverse problems," Phys. Med. Biol., vol. 32, pp. 11-22, 1987.

[46] H. A. Schlitt, L. Heller, R. Aaron, E. Best, and D. M. Ranken, "Evaluation of boundary element method for the EEG forward problem: Effect of linear interpolation," IEEE Trans. Biomed. Eng., vol. 42, pp. 52-57, 1995.

[47] R. Srebro, "A modified boundary element method for the estimation of potential fields on the scalp," IEEE Trans. Biomed Eng., vol. 43. pp. 650-653, June 1996.

[48] G. Strang and G. J. Fix, An Analysis of the Finite Element Method Englewood Cliffs, NJ: Prentice-Hall, 1973.

[49] J. Tripp, "Physical concepts and mathematical models," Biomagnetism. An Interdisciplinary Approach, S. J. Williamson, G. L. Romani, L. Kaufman, and I. Modena, Eds. New York: Plenum, 1983, pp. 101-139. 
[50] L. Urankar, "Common compact analytical formulas for computation of geometry integrals on a basic Cartesian subdomain in boundary and volume integrals," Eng. Anal. with Boundary Elements, vol. 7, no. 3, pp. 124-129, 1990.

[51] J. Wikswo, "SQUID magnetometers for biomagnetism and nondestructive testing: important questions and initial answers," IEEE Trans. Appl. Superconduct., 1995 , pp. 74-120.

[52] Z. Zhang, "A fast method to compute surface potentials generated by dipoles within multilayer anisotropic spheres," Phys. Med. Biol., vol. 40, pp. 335-349, Mar. 1995.

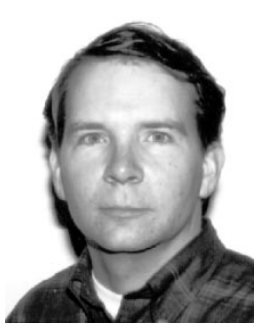

John C. Mosher received the B.S. degree in electrical engineering with highest honors from the Georgia Institute of Technology, Atlanta, in 1983. From 1979-1983, he was also a cooperative education student with Hughes Aircraft Company Fullerton, CA. He received the M.S. and Ph.D. degrees in electrical engineering from the Signal and Image Processing Institute of the University of Southern California (USC), Los Angeles, in 1985 and 1993, respectively.

From 1983-1993, he worked at TRW in Los Angeles, CA, researching signal analysis procedures for electromagnetic pulse effects. Upon graduation, he accepted a staff position at the Los Alamos National Laboratory, Los Alamos, NM, where he researches the forward and inverse modeling problems of electrophysiological recordings. He is a member of the Design Technology Group. His interests also include the general source localization and imaging problems, both in neuroscience work and in other novel applications of sensor technology.

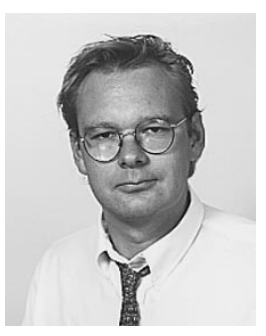

Richard Leahy was born in Surrey, U.K., in 1960. He received the B.Sc. and Ph.D. degrees in electrical engineering from the University of Newcastle upon Tyne, U.K., in 1981 and 1985, respectively.

In 1985, he joined the University of Southern California (USC), where he is currently a Professor in the Department of Electrical Engineering-Systems and Director of the Signal and Image Processing Institute. He holds joint appointments with the Departments of Radiology and Biomedical Engineering at USC. His research interests lie in the application of signal and image processing theory to biomedical inverse problems. His current research involves the reconstruction and analysis of medical images with an emphasis on PET, MEG, and MRI.

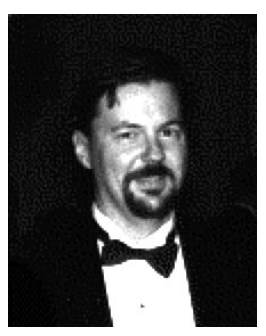

Paul S. Lewis was born in Albany, NY, on January 22, 1956. He received the B.S. degree in electrical engineering from the Massachusetts Institute of Technology, Cambridge, in 1978, the M.S. degree in electrical engineering from the University of New Mexico, Albuquerque, in 1984, and the Ph.D. degree in electrical engineering from the University of Southern California, Los Angeles, in 1988.

He has been a Staff Member at the Los Alamos National Laboratory, Los Alamos, NM, since 1978 His current work is in the area of digital signal processing and involves research in adaptive algorithms, parallel computer architectures, and biomedical applications. 